\title{
Respuesta fisiológica y química de clones de Ulmus minor susceptibles y resistentes a la grafiosis tras la inoculación con Ophiostoma novo-ulmi
}

\author{
Rodríguez-Calcerrada, J., ${ }^{1 *}$ Li, M., ${ }^{1}$ López, R., ${ }^{1}$ Venturas, M., ${ }^{1}$ Martín, J.A., ${ }^{1}$ \\ Domínguez, J., Gordaliza, G.G., ${ }^{1}$ Gil, L. ${ }^{1}$ \\ ${ }^{1}$ Grupo de Investigación en Genética y Fisiología Forestal, ETSI Montes, Universidad Politécnica de Madrid. \\ Ciudad Universitaria s/ $n$, 28040 Madrid (España) \\ *Autor para correspondencia: jesus.rcalcerrada@upm.es
}

\begin{abstract}
Resumen
Los motivos por los que algunos genotipos de Ulmus minor Mill. resisten más que otros a la infección con el hongo patógeno Ophiostoma novo-ulmi son aun desconocidos. Con el objetivo de evaluar si la resistencia a la enfermedad de la grafiosis está relacionada con la posesión de ciertos rasgos fisiológicos o químicos, se compararon clones de U. minor resistentes y susceptibles a la grafiosis, antes y después de la inoculación con O. novo-ulmi. Se midieron el potencial hídrico, las tasas de respiración y fotosíntesis foliar, y la conductividad hidráulica de ramas terminales y su composición química mediante espectroscopía de infrarrojo (FT-IR). La inoculación con el hongo produjo un aumento en la proporción de vasos embolizados, de modo que a los 21 días la conductividad hidráulica era solo un $20 \%$ de la conductividad máxima en los clones susceptibles. Como consecuencia, el potencial hídrico y la fotosíntesis disminuyeron entorno a un 100-200\% en relación a los controles en los clones susceptibles mientras que no hubo reducciones significativas en los resistentes. Además, los clones mostraron una composición química de sus ramas diferente. Por ejemplo, en los árboles inoculados con agua utilizados como control, el pico de absorción en la región del espectro infrarrojo relacionado con la suberina fue más alto en los clones resistentes que en los susceptibles. Estos resultados sugieren que el perfil químico más defensivo de los clones resistentes les permite mantener la funcionalidad fisiológica tras la inoculación con $O$. novo-ulmi prácticamente inalterada, en comparación con los clones más susceptibles.
\end{abstract}

Palabras clave: plaga, olmo, decaimiento, sanidad, cavitación, defensa. 


\section{Introducción}

La lucha contra la enfermedad de la grafiosis, que continúa diezmando las poblaciones y ejemplares aislados de olmo desde hace casi 100 años en España, se centra hoy en día en la detección y propagación de clones de olmo resistentes. En los últimos años, dentro del Programa Español de Conservación y Mejora de los Olmos Ibéricos que desarrollan el Ministerio de Agricultura, Alimentación y Medio Ambiente y la Universidad Politécnica de Madrid, se ha examinado la resistencia de miles de genotipos de olmo común (Ulmus minor Mill; olmo de aquí en adelante) a la inoculación controlada de cepas virulentas de Ophiostoma novo-ulmi. Los genotipos que superan la inoculación con el patógeno durante dos años consecutivos sufriendo marchitamientos inferiores al $30 \%$ de su copa y que, además, satisfacen una serie de requisitos estéticos y de crecimiento (i.e. copas esbeltas y redondeadas y tasas de crecimiento considerables) son seleccionados como olmos resistentes. Gracias al esfuerzo continuo desde 1986, en 2014 (BOE-A-2014-1353) se incorporaron siete genotipos de olmo resistentes al Catálogo Nacional de Materiales de Base para la producción de material forestal de reproducción cualificado (MAGRAMA, 2015).

En paralelo a esta línea de actuación es necesario averiguar las causas de la resistencia a la enfermedad de la grafiosis para mejorar los procesos de selección de individuos resistentes y de esta manera apoyar la conservación de los olmos a nivel mundial. No obstante, se desconoce en gran medida qué rasgos confieren a unos pocos genotipos la capacidad de enfrentarse a $O$. novo-ulmi sin sufrir apenas daños. Se ha indicado que genotipos con vasos xilemáticos de pequeño tamaño y en su mayoría desagrupados son menos susceptibles a sufrir daños por $O$. novo-ulmi que aquellos con vasos agrupados y de mayor tamaño (Solla y Gil, 2002). En este sentido, se ha sugerido que los olmos cuyo periodo de crecimiento primaveral se inicia relativamente temprano podrían ser menos susceptibles a la grafiosis que aquellos que crecen más adelante, ya que estos últimos forman la madera temprana (de vasos de mayor tamaño) en el momento de máxima actividad de los insectos escolítidos que transportan las esporas de $O$. novo-ulmi, mientras que los primeros ya habrían iniciado la formación de vasos más pequeños, más resistentes a la cavitación (Ghelardini y Santini, 2009). La constitución química de los tallos podría también tener un papel importante en la resistencia a la grafiosis. Los trabajos realizados por Martin et al. $(2005,2008)$ sugieren que los tallos de los genotipos más resistentes a la inoculación con $O$. novo-ulmi contienen más lignina, suberina, almidón, celulosa y pectina que aquellos más susceptibles a la enfermedad. Dado que estos compuestos participan de un modo directo o indirecto en la defensa del árbol frente a diversos agentes patógenos, Martin et al. $(2005,2008)$ sugirieron la relación entre el perfil químico y la resistencia a la grafiosis de algunos genotipos de olmo.

En comparación con la evaluación de rasgos químicos y anatómicos, la evaluación de rasgos fisiológicos ha tenido un peso menor en los estudios sobre la grafiosis de los últimos años. Destacan los trabajos de Oliveira et al., (2012), Urban y 
Dvoŕák (2014) y Venturas et al., (2014); en ellos se examinaba el impacto de la inoculación con $O$. novo-ulmi en la conductividad hidráulica y flujo de savia bruta de los tallos, así como la capacidad de las hojas para asimilar dióxido de carbono $\left(\mathrm{CO}_{2}\right)$. Todos estos procesos se verían mermados por la propagación del hongo que, a medida que coloniza el árbol, causa la cavitación y oclusión de los vasos xilemáticos limitando el transporte de agua a las hojas, que pierden turgencia y capacidad fotosintética y, finalmente, acaban marchitándose en las ramas afectadas.

No obstante, se precisan más trabajos sobre el grado y tipo de disfuncionalidad que causa la inoculación con $O$. novo-ulmi en clones de olmo de resistencia al patógeno contrastada para comprender qué factores determinan dicha resistencia. En este sentido, la disponibilidad de genotipos altamente resistentes ofrece una oportunidad única para desentrañar las causas de la resistencia a la grafiosis.

En este trabajo se presentan los resultados de un experimento realizado en el Centro Nacional de Recursos Genéticos Forestales "Puerta de Hierro" (Madrid) en el que se ha comparado el perfil químico y fisiológico de dos clones previamente identificados como resistentes o susceptibles a la grafiosis. El objetivo general fue identificar rasgos fisiológicos y químicos que pudieran estar involucrados en la resistencia frente al patógeno causante de la grafiosis. La hipótesis de trabajo fue que los clones resistentes mostrarían un perfil químico más propicio para la defensa frente a $O$. novo-ulmi, y por tanto un menor grado de afección en las variables fisiológicas que los clones susceptibles. Para explorar esta hipótesis se midió la conductividad hidráulica y composición química de ramas, y el potencial hídrico y tasa de intercambio gaseoso foliar en 4 individuos por clon antes y después de ser inoculados con O. novo-ulmi.

\section{Material y métodos}

\subsection{Material vegetal y diseño experimental}

Se compararon los clones de olmo resistentes AB-AM2.4 y M-DV2.3 con los clones susceptibles VA-AP38 y M-DV1. Los individuos de los clones AB-AM2.4, M-DV2.3 y M-DV1 tenían 5 años de edad en el momento de la inoculación, mientras que los del clon VA-AP38 tenían 6 años.

Los árboles estaban plantados en terrenos llanos y desprovistos de vegetación herbácea, sin limitaciones hídricas debido al aporte de agua desde el momento de su plantación. Se evitó la posible interacción de factores ambientales desconocidos en el crecimiento de los árboles (y por tanto en el resultado de las inoculaciones) gracias a la plantación de los individuos por bloques, en un marco de $1 \times 1 \mathrm{~m}$.

Dos árboles de cada genotipo se inocularon en la primera semana de Mayo de 2013 con $0.1 \mathrm{ml}$ de una suspensión de 106 esporas ml-1 de la cepa O. novo-ulmi Brasier ssp. americana Z-BU1. La inoculación se realizó aplicando la suspensión de esporas contenida en una jeringa a un corte transversal de aproximadamente 2$4 \mathrm{~mm}$ de profundidad realizado en el tallo principal a unos 10-15 $\mathrm{cm}$ del suelo. La 
mitad de los árboles se inocularon con agua destilada libre de esporas de hongo para disponer de controles. En ambos casos se hicieron las inoculaciones en días soleados para asegurar la absorción de la solución en el flujo transpiratorio del árbol.

El muestreo para los análisis químicos y fisiológicos se realizó en varios momentos a lo largo de 21 días tras la inoculación. Según el estado de marchitamiento de los árboles se seleccionaron hojas y ramas visualmente sanas (cuando menos del $50 \%$ de las hojas amarilleaban) o cloróticas (cuando más del 50\% de las hojas amarilleaban). La elección de este muestreo no aleatorio se debió al efecto de la infección por $O$. novo-ulmi, que afecta de manera muy desigual a las ramas del árbol (ver Domec et al. 2013 para la aplicación de un muestreo similar en relación a la propagación de Adelges tsugae).

\subsection{Potencial hibrido}

Se midió el potencial hídrico de hojas de la parte baja de la copa con una cámara de presión de tipo Schölander (PMS Instrument Company, Albany, EEUU), tanto al amanecer como a mediodía, como indicador del estado hídrico de los árboles.

\subsection{Intercambio gaseoso foliar}

Se midió el intercambio de vapor de agua y $\mathrm{CO}_{2}$ en hojas de la parte baja de la copa con un equipo de análisis de gases por infrarrojo (IRGA, de sus siglas en inglés; Li-Cor Inc., EEUU). Las medidas de conductancia estomática y asimilación neta de $\mathrm{CO}_{2}$ se realizaron entre las 10.00 y las $13.00 \mathrm{~h}^{2} 800 \mathrm{mmol} \mathrm{m}^{-2} \mathrm{~s}^{-1}$ de luz, $400 \mathrm{ppm}$ de concentración de $\mathrm{CO}_{2}$ y $25^{\circ} \mathrm{C}$ de temperatura del aire. Las de respiración se realizaron posteriormente también a $400 \mathrm{ppm}$ de concentración de $\mathrm{CO}_{2} \mathrm{y}$ una temperatura similar en condiciones de oscuridad mantenida durante $30 \mathrm{minu}$ tos.

\subsection{Conductancia hidráulica}

Se midió en ramas de la parte superior de la copa con un equipo de medición de embolia XYL'EM (Bronkhorst High-Tech B.V., Francia). Las ramas se cortaron tras sumergir la zona de corte en un balde con agua. Ya en el laboratorio, las ramas se recortaron de nuevo y se descortezaron bajo agua. La conductividad hidráulica inicial (k) se midió haciendo pasar por el xilema del ramillo una solución de agua desgasificada $10 \mathrm{mM} \mathrm{KCl}$ y $1 \mathrm{mM} \mathrm{CaCl}_{2}$ a $2-310^{-3} \mathrm{MPa}$ de presión. La conductividad hidráulica máxima $\left(\mathrm{k}_{\max }\right)$ se midió del mismo modo tras hacer pasar dicha solución por el xilema del ramillo a $0.18 \mathrm{MPa}$ durante $10 \mathrm{~min}$. En función de estas variables se calculó la pérdida de conductividad hidráulica del xilema relativa a la máxima capacidad (PLC, de sus siglas en inglés) con la ecuación: PLC $=100\left(\mathrm{k}_{\max }-\mathrm{k}\right) / \mathrm{k}_{\max }$. 
Los valores de $\mathrm{k}$ y kmax se estandarizaron dividiéndolos por el área de la sección transversal del xilema para obtener $\mathrm{k}_{\mathrm{s}} \mathrm{y} \mathrm{k}_{\mathrm{smax}}$, respectivamente.

\subsection{Composición química}

Se evaluó a través del análisis por espectroscopía infrarroja por transformada de Fourier (FT-IR) de ramillos con un equipo Perkin-Elmer 1600 (Perkin Elmer Inc, EEUU). Los ramillos (1-1,5 cm de diámetro) se cortaron y se sumergieron en un contenedor con nitrógeno líquido por unas horas hasta su almacenamiento a $-80^{\circ} \mathrm{C}$. Las ramas congeladas se trocearon y posteriormente se liofilizaron y trituraron hasta alcanzar un polvo fino. La mezcla de $3 \mathrm{mg}$ de este polvo con $240 \mathrm{mg}$ de $\mathrm{KBr}$ se prensó al vacío a $10 \times 10^{4} \mathrm{~N}$, obteniéndose una pastilla fina translúcida que fue la que finalmente se analizó en el equipo. Por cada muestra se obtuvieron tres espectros de absorción entre 600 y $4000 \mathrm{~cm}^{-1}$ (acumulando 64 interferogramas a una resolución de $4 \mathrm{~cm}^{-1}$ ) y se calculó la media. De cada espectro se midió la altura de los 19 picos de absorción principales como variables relacionadas con el perfil químico de las ramas.

\subsection{Análisis estadístico}

La significación estadística de las diferencias entre los valores medios de los dos tratamientos (árboles inoculados con agua o con $O$. novo-ulmi) y de los dos grupos de olmos (resistentes y susceptibles a priori) se evaluó con el test t de Student, o el test Welch cuando las varianzas eran desiguales.

\section{Resultados}

\subsection{Marchitamiento}

Los individuos de los clones susceptibles empezaron a mostrar ramas con hojas marchitas tras 14 días de ser inoculados con $O$. novo-ulmi; tras 21 días aproximadamente el $60 \%$ de las hojas estaban marchitas o secas. En el caso de los individuos de los clones resistentes, el porcentaje de marchitamiento medio fue del $7.5 \%$ en el clon AB-AM2.4 y del 15\% en el clon M-DV2.3 tras 21 días desde la inoculación (Fig. 1).

\subsection{Variables fisiológicas}

No se apreciaron diferencias significativas entre clones resistentes y susceptibles en ninguna de las variables fisiológicas previamente a la inoculación. Al contrario, en respuesta a la inoculación, sí se apreciaron diferencias en el comportamiento de los clones: en el caso de los clones resistentes, el potencial hídrico foliar, las tasas 


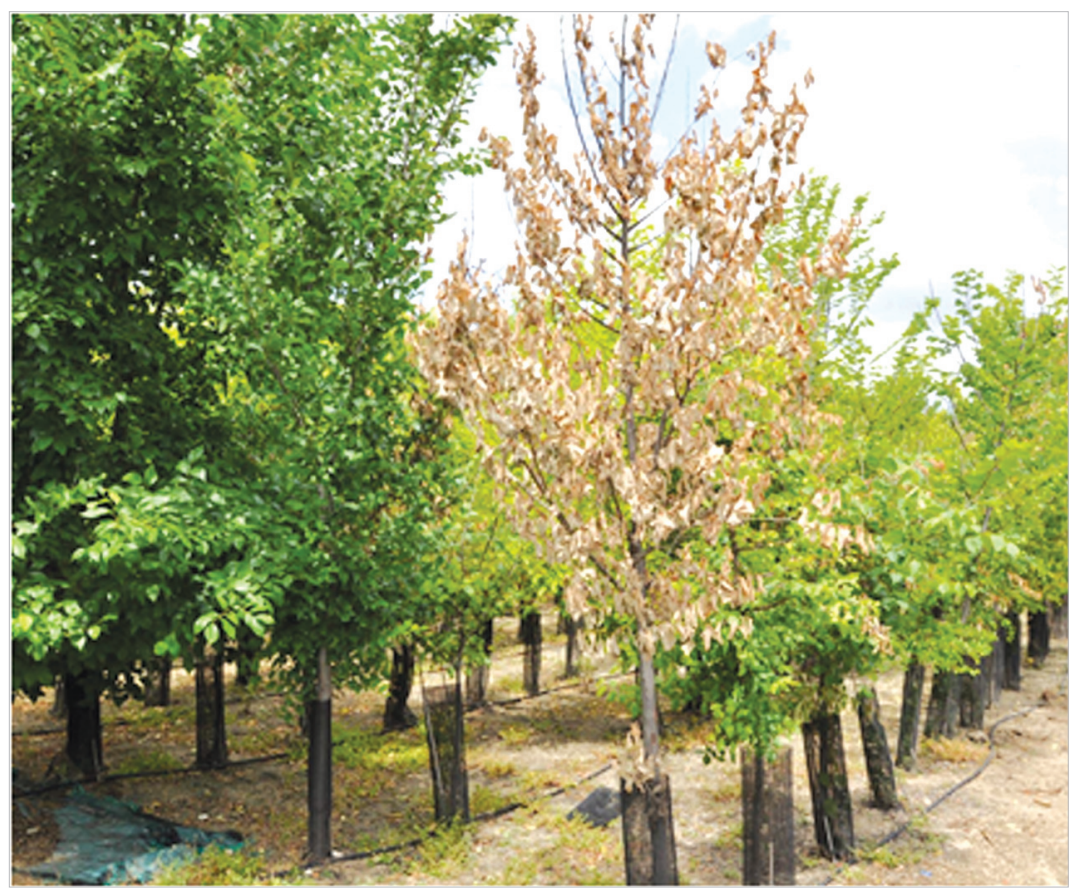

Figura 1. Árboles de clones susceptibles con la mayoría de sus ramas secas (derecha) y clones resistentes sin signos de daño (izquierda) tras la inoculación con O. novo-ulmi.

de conductancia estomática y asimilación neta de $\mathrm{CO}_{2}$, y la conductividad hidráulica fueron similares en los individuos inoculados con agua y O. novo-ulmi (Fig. 2). Sin embargo, en el caso de los clones susceptibles, todas estas variables difirieron significativamente entre ambos tratamientos tras 21 días desde la inoculación; igualmente, la pérdida de conductividad hidráulica media (no mostrada en ninguna figura) superó el $80 \%$ en los individuos inoculados con O. novo-ulmi y no llegó al $40 \%$ en los inoculados con agua (Fig. 2).

\subsection{Variables químicas}

Existieron pequeñas diferencias en algunos picos de absorción entre clones resistentes y susceptibles previamente a la inoculación. La inoculación con O. novo-ulmi tuvo un efecto poco marcado y no se observaron diferencias químicas significativas entre los individuos control e inoculados con O. novo-ulmi, ni en los clones resistentes ni en los susceptibles. Por este motivo se decidió agrupar ambos tratamientos y comparar los clones tras 21 días desde la inoculación. Dicha comparación sí evidenció diferencias entre clones: el pico de absorción a $2922 \mathrm{~cm}^{-1}$, relacionado con hidrocarburos saturados como la suberina y los ácidos grasos (Pandey y Pitman, 2003) fue más alto en los clones resistentes que en los susceptibles. Igualmente existieron diferencias en los picos de absorción a 1374, 1248 y $888 \mathrm{~cm}^{-1}$, relacionados con el contenido de celulosa (Pandey y Pitman, 2003), compuestos fenólicos (Sene et al., 1994), y pectina (Pandey y Pitman, 2003), respectivamente, siendo todos ellos también más altos en los clones resistentes que en los susceptibles (Fig. 3). 

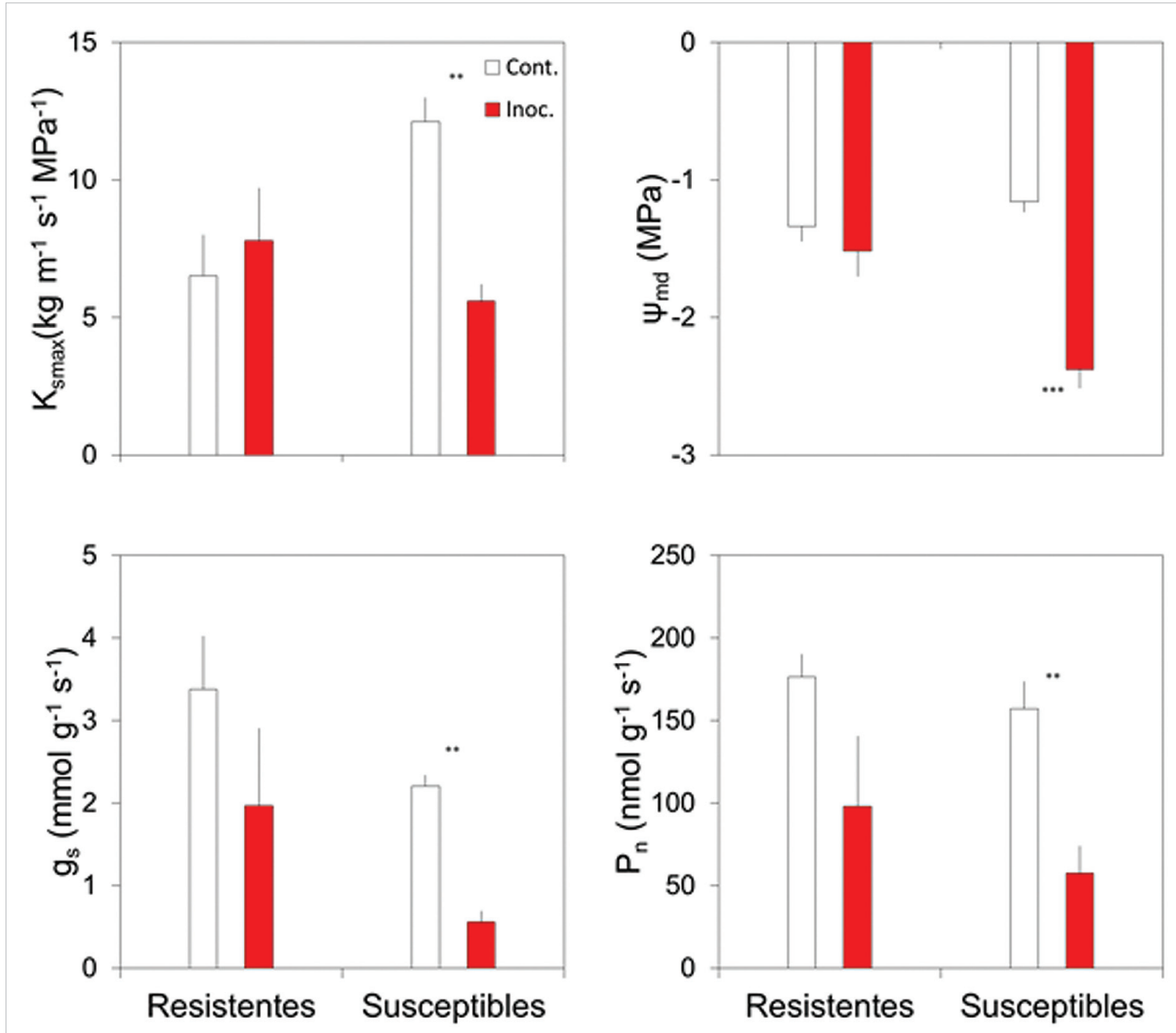

Figura 2. Comparación de los valores medios (y error estándar) de diversas variables fisiológicas en árboles de clones resistentes y susceptibles inoculados con agua (controles, barras blancas) u O. novo-ulmi (barras rojas). Los asteriscos indican el nivel de significación estadística de pruebas $t$-Student (**: P-valor < 0.01 ; ***: P-valor $<0.001)$. Abreviaciones: $\mathrm{K}_{\text {mmax }}$ conductividad hidráulica máxima específica; $\Psi_{\operatorname{md}}:$ potencial hídrico foliar medido a mediodía; gs: conductancia estomática al vapor de agua; $\mathrm{P}_{\mathrm{n}}$ : asimilación fotosintética neta de $\mathrm{CO}_{2}$.

\section{Discusión}

Los esfuerzos de lucha contra la grafiosis se plasmaron en 2014 en la catalogación de siete genotipos de olmo resistentes (BOE-A-2014-1353; Martín et al. 2015). Uno de ellos es el clon de Madrid (procedente del parque de la Dehesa de la Villa) M-DV2.3, examinado en este estudio junto al clon resistente de Albacete AB-AM2.4. Sometidos a la inoculación con $O$. novo-ulmi, ambos clones apenas vieron mermada la funcionalidad de sus ramas y hojas, mientras que los clones M-DV1 y VA-AP38 mostraron una elevada susceptibilidad a la inoculación, sufrieron una clara pérdida de conductividad hidráulica en sus ramas, y la caída del potencial hídrico y las tasas de asimilación neta de $\mathrm{CO}_{2}$. Esta respuesta diferencial es reflejo, pero también causa 

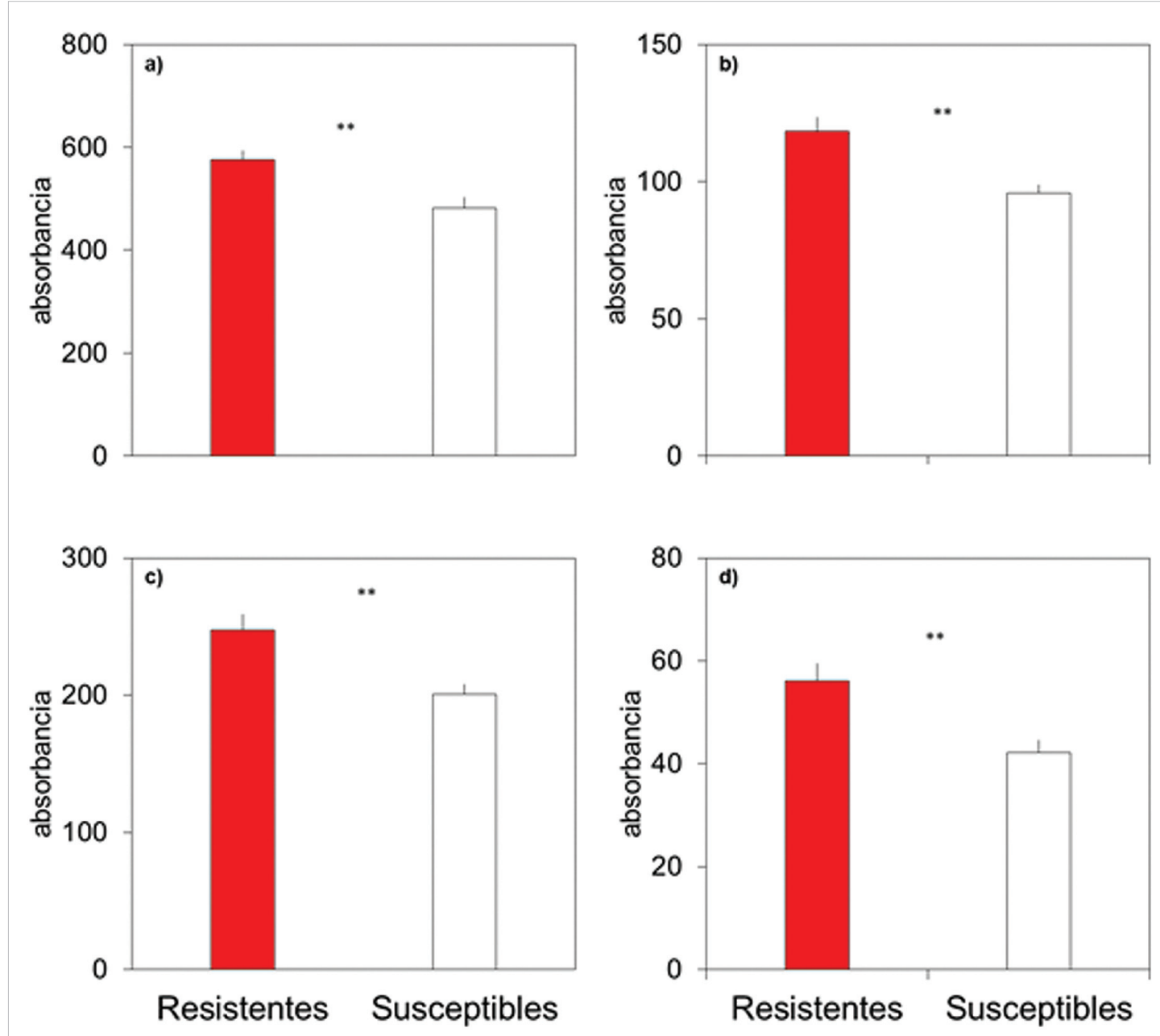

Figura 3. Comparación de los valores medios (y error estándar) de varios picos de absorción en el espectro infrarrojo a distintas longitudes de onda [a), $2922 \mathrm{~cm}^{-1}$, relacionado con la suberina; b) $1374 \mathrm{~cm}^{-1}$ relacionado con la celulosa; c) $1248 \mathrm{~cm}^{-1}$ relacionado con los fenoles; d) $888 \mathrm{~cm}^{-1}$ relacionado con la celulosa y la pectina] en árboles de clones resistentes (barras rojas) y susceptibles (barras blancas) - agrupando individuos inoculados con agua y $O$. novo-ulmi dado que entre estos dos tratamientos no se detectaron diferencias significativas tras 21 días desde la inoculación. Los asteriscos indican el nivel de significación estadística de pruebas $t$-Student (**: P-valor $<0.01)$.

de la diferente susceptibilidad entre clones al patógeno. Así, el hecho de que los árboles mantengan casi intacta su capacidad de transporte de agua y asimilación de $\mathrm{CO}_{2}$ contribuye a que puedan seguir sintetizando azúcares necesarios para la producción de compuestos defensivos, el crecimiento y/o el rebrote en caso de que algunas de sus ramas se sequen bien sea por la propia enfermedad o por daños asociados a sequías u otro tipo de factores de estrés. Se pensó inicialmente que los clones resistentes podrían tener algunas características fisiológicas intrínsecas que los diferenciaran de los susceptibles, como sucede con algunas características anatómicas (Solla y Gil 2002). Sin embargo, no se detectaron diferencias constitutivas entre clones en ninguna de las variables hidráulicas o relacionadas con el intercambio ga- 
seoso. Esto no excluye que existan diferencias fisiológicas en otro tipo de características, o incluso en las mismas que se han examinado pero a otros niveles, por ejemplo al nivel de árbol en lugar de órgano en el caso de la transpiración.

La grafiosis es una enfermedad vascular. En este trabajo se pone de manifiesto que la propagación de $O$. novo-ulmi causa una marcada reducción del transporte de savia bruta en las ramas terminales pocas semanas después de su inoculación en la base del tronco. Estas limitaciones en el transporte de savia son debidas en parte al propio bloqueo que genera en los vasos xilemáticos el crecimiento de las hifas del hongo y otras sustancias o estructuras del árbol (e.g., gomas y tilosas) emitidas en respuesta a la presencia del hongo. Este hecho se pone de manifiesto en la Fig. 2. En ella se puede apreciar que la conductividad hidráulica máxima, obtenida después de hacer pasar agua desgasificada a alta presión, y por tanto rellenar supuestamente los vasos embolizados, es la mitad en los árboles inoculados con O. novo-ulmi que en los árboles de control, en el caso de los clones susceptibles. La consecuencia del bloqueo de los vasos es la brusca caída del potencial hídrico de las hojas y de su capacidad de asimilación de $\mathrm{CO}_{2}$. Sin embargo, a pesar de que el estrés hídrico suele causar una reducción de la respiración mitocondrial (e.g. Rodríguez-Calcerrada et al. 2011), no se observó una reducción de la tasa de respiración en los árboles inoculados con O. novo-ulmi; al contrario, dicha tasa aumentó significativamente con relación a los árboles de control, posiblemente debido al pico de respiración que se asocia en algunas ocasiones a la senescencia foliar y el reciclaje de nutrientes de las hojas cloróticas que están a punto de secarse.

Mientras que la sucesión de alteraciones fisiológicas que causa $O$. novo-ulmi en los árboles susceptibles está más o menos clara, los motivos por los que ésta no llega a impedir el transporte de agua y por consiguiente alterar el intercambio gaseoso foliar en los genotipos resistentes M-DV2.3 y AB-AM2.4 no están claros. Los resultados obtenidos a través del análisis de espectroscopía infrarroja sugieren que la posesión de niveles más elevados de ciertos compuestos en los clones resistentes limita la propagación del hongo por el árbol. Tal es el caso de la suberina, que es resistente a la degradación por parte del hongo impidiendo su expansión, y los compuestos fenólicos, que tienen actividad antimicrobiana y participan en la señalización y regulación de importantes rutas metabólicas de defensa (Witzell and Martín 2008). La posesión de niveles más elevados de otros compuestos como la celulosa en los clones resistentes de olmo también podría relacionarse directamente con la resistencia al patógeno por su papel en la síntesis de la pared celular, y la ventaja que constituiría el refuerzo de la pared al dificultar el ataque de hongos (FurstenbergHagg et al., 2013).

Cabe mencionar dos hechos más en relación al perfil químico. Uno es que los árboles inoculados con agua y $O$. novo-ulmi apenas mostraran diferencias en su composición química en ninguno de los clones. El otro es que las diferencias entre clones fueran más marcadas a los 21 días que al inicio del experimento. En relación con este último hecho se sugiere que las heridas provocadas por el muestreo de las ramas para la caracterización química e hidráulica, o por la propia inoculación del hongo o del agua, indujeron la síntesis de compuestos químicos defensivos, tales 
como la suberina u otro tipo de compuestos fenólicos. La inducción de vías metabólicas de defensa por heridas es un hecho constatado en muchas especies vegetales (e.g. León et al., 2001); sin embargo, esta posibilidad no se había sugerido antes en el caso de la grafiosis y requiere de experimentos futuros para contrastarla.

\section{Conclusiones}

El perfil químico más defensivo de los genotipos resistentes de olmo limita la propagación de $O$. novo-ulmi y los daños funcionales asociados que causa este patógeno: pérdida de conductividad hidráulica por el bloqueo y cavitación de los vasos xilemáticos y, finalmente, marchitamiento de hojas y secado de ramas.

\section{Agradecimientos}

Agradecemos la colaboración de Paz Andrés en los análisis químicos y la discusión científica de los datos con Pedro Perdiguero y Carmen Collada. También agradecemos el apoyo de la Dirección General de Política Forestal y Desarrollo Rural (Ministerio de Agricultura, Alimentación y Medio Ambiente) y la financiación del Ministerio de Economía y Competitividad a través del proyecto OLMOS (AGL2012-35580).

\section{Bibliografía}

Domec, J.C., Rivera, L.N., King, J.S., Peszlen, I., Hain, F., Smith, B., Frampton, J., 2013. Hemlock woolly adelgid (Adelges tsugae) infestation affects water and carbon relations of eastern hemlock (Tsuga canadensis) and Carolina hemlock (Tsuga caroliniana). New Phytol 199, 452-463.

Furstenberg-Hagg, J., Zagrobelny, M., Bak, S., 2013. Plant Defense against Insect Herbivores. Int. J. Mol. Sci. 14, 10242-10297.

Ghelardini, L., Santini, A., 2009. Avoidance by early flushing: a new perspective on Dutch elm disease research. iForest 2, 143-153.

León, J., Rojo, E., Sánchez-Serrano, J.J., 2001. Wound signalling in plants. J. Exp. Bot. 52, 1-9.

MAGRAMA, 2015. http://www.magrama.gob.es/es/desarrollo-rural/temas/politica-forestal/ recursos-geneticos-forestales/rgf_catalogo_materiales_base_reprod_culif.aspx. Último acceso: 3 Noviembre 2015.

Martín, J.A., Solla, A., Woodward, S., Gil, L., 2005. Fourier transform-infrared spectroscopy as a new method for evaluating host resistance in the Dutch elm disease complex. Tree Physiol. 25, 1331-1338.

Martín, J.A., Solla, A., Coimbra, M.A., Gil, L., 2008. Metabolic fingerprinting allows discrimination between Ulmus pumila and U. minor, and between $U$. minor clones of different susceptibility to Dutch elm disease. For Pathol 38, 244-256. 
Martin, J.A., Solla, A., Venturas, M., Collada, C.J., Miranda, E., Fuentes, P., Burón, M., Iglesias, S., Gil, L. 2015. Seven Ulmus minor clones tolerant to Ophiostoma novo-ulmi registered as forest reproductive material in Spain. iForest 8, 172-180.

Oliveira, H., Sousa, A., Alves, A., Nogueira, A. J. A., Santos, C., 2012. Inoculation with Ophiostoma novo-ulmi subsp. americana affects photosynthesis, nutrition and oxidative stress in in vitro Ulmus minor plants. Environ. Exp. Bot. 77, 146-155.

Pandey, K.K., Pitman, A.J., 2003. FTIR studies of the changes in wood chemistry following decay by brown-rot and white-rot fungi. Int. Biodeterior. Biodegrad. 52, 151-160.

Rodríguez-Calcerrada, J., Shahin, O., del Rey, M.C., Rambal, S., 2011. Opposite changes in leaf dark respiration and soluble sugars with drought in two Mediterranean oaks. Funct. Plant Biol. 38, 1004-1015.

Sene, C.F., McCann, M.C., Wilson, R.H., Grinter, R., 1994. Fourier-transform Raman and Fourier-transform infrared spectroscopy (an investigation of five higher plant cell walls and their components). Plant Physiol. 106, 1623-1631.

Solla, A., Gil, L., 2002. Xylem vessel diameter as a factor in resistance of Ulmus minor to Ophiostoma novo-ulmi. For. Pathol. 32, 123-134.

Urban, J., Dvořák, M., 2014. Sap flow-based quantitative indication of progression of Dutch elm disease after inoculation with Ophiostoma novo-ulmi. Trees 28, 1599-1605.

Venturas, M., López, R., Martín, J., Gascó, A., Gil, L., 2014. Heritability of Ulmus minor resistance to Dutch elm disease and its relationship to vessel size, but not to xylem vulnerability to drought. Plant Pathol. 63, 500-509.

Witzell, J., Martín, J.A., 2008. Phenolic metabolites in the resistance of northern forest trees to pathogens - past experiences and future prospects. Can. J. For. Res. 38, 2711-2727. 
